\title{
The active role of methanol in post-synthetic linker exchange in the metal-organic framework UiO-66
}

\author{
Joao Marreirosł, Chiara Caratellił, Julianna Hajekł, Andraž Krajnc§, Guillaume \\ Fleury†, Bart Bueken†, Dirk E. De Vost, Gregor Mali§, Maarten B.J. Roeffaerst, Ve- \\ ronique Van Speybroeck , Rob Ameloot†*
}

tCentre for Surface Chemistry and Catalysis, KU Leuven, Celestijnenlaan 200F p.o. box 2461, 3001 Leuven, Belgium

‡Center for Molecular Modeling, Universiteit Gent, Technologiepark 903, B-9052 Zwijnaarde, Belgium

§Department of Inorganic Chemistry and Technology, National Institute of Chemistry, Hajdrihova 19, 1001 Ljubljana, Slovenia

\begin{abstract}
UiO-66 is known as one of the most robust metal-organic framework materials. Nevertheless, UiO-66 has also been shown to undergo post-synthetic exchange of structural linkers with surprising ease in some solvents. To date, the exchange mechanism has not yet been fully elucidated. Here, we show how timeresolved monitoring grants insight into the selected case of exchanging 2-aminoterephthalate into UiO-66 in methanol. Analysis of both the solid and liquid phase, complemented by computational insights, revealed the active role of methanol in the creation and stabilization of dangling linkers. Similar to monocarboxylate defects that can be introduced during UiO-66 synthesis, such dangling linkers undergo fast exchange. The presence of missing linker or missing cluster defects at the start of the exchange process was shown to have no considerable impact on the equilibrium composition. After the exchange process, the incoming 2aminoterephthalate and remaining terephthalate linkers were distributed homogeneously in the framework for the typical sub-micron size of UiO-66 crystallites.
\end{abstract}

Metal-organic frameworks (MOFs) are porous crystalline materials currently at the forefront of materials chemistry due to their high internal surface area and tunable nature. MOFs are constructed from metal ion nodes connected by multitopic organic linkers. One of the most promising MOF materials, due to its high stability, is the widely studied UiO-66, comprised of 12coordinated $\left[\mathrm{Zr}_{6}\left(\mu_{3}-\mathrm{O}\right)_{4}\left(\mu_{3}-\mathrm{OH}\right)_{4}\right]^{12+} \quad$ clusters connected through 1,4-benzenedicarboxylate (BDC) linkers. ${ }^{1}$ UiO-66 presents high framework connectivity combined with strong coordination bonds between the oxophilic $\mathrm{Zr}^{4+}$-based clusters and the BDC carboxylate groups. ${ }^{2}$ Moreover, because of its high connectivity, the UiO-66 framework has a high tolerance for structural defects, ${ }^{3}$ a property that has been explored in catalysis, 4,5 gas storage and separation, ${ }^{6,7}$ sensing, ${ }^{8}$ drug delivery, ${ }^{9}$ etc. Despite their presumed robust and inert nature, UiO-66 and other Zr-MOFs have been found to be susceptible to a range of post-synthetic modification (PSM) processes. For instance, Farha and coworkers reported the post-synthetic grafting of linkers onto coordinatively unsaturated sites in the MOF material NU-100o. ${ }^{10,11} \mathrm{Kim}$ et al. demonstrated that the BDC linker in UiO-66 can be exchanged with a differently functionalized analogue without a loss in crystallinity, in a process termed 'post-synthetic ligand exchange' (PSE, Figure 1). ${ }^{12}$

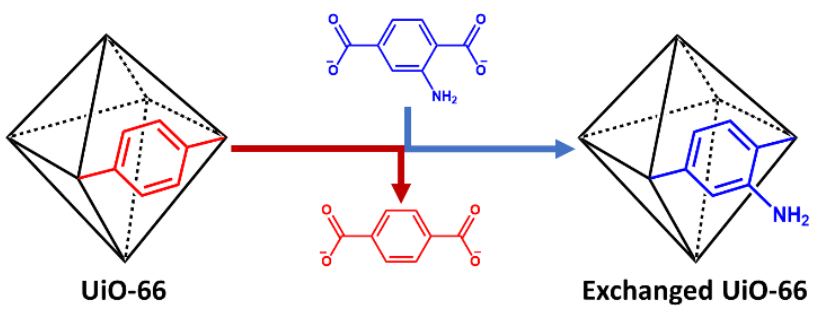

Figure 1. PSE in UiO-66. The structural linker in the framework is replaced and released into the surrounding solution.

Since its introduction, PSE has become a wellestablished procedure in the preparation of UiO-66 materials with specific chemical functionalities, often inaccessible through direct synthesis ${ }^{13,14}$. Nevertheless, a fundamental understanding of the PSE process is still lacking. Furthermore, different reported exchange protocols lead to seemingly contradictory results, in terms of the extent of linker exchange and distribution. Recently, a number of studies have demonstrated how the particle size can impact whether or not the incoming linker will be distributed homogeneously in the modified 
framework. ${ }^{15,14}$ Shearer et al. and Taddei et al. demonstrated how defect sites are more prone to exchange than the structural linker of the parent framework. ${ }^{16,17}$ Both reports show that when performed in DMF, incoming monodentate ligands or bidentate linkers end up predominantly at defect sites capped by monocarboxylates. The latter are often introduced during synthesis through the use of 'modulators' and are more easily displaced than the doubly anchored dicarboxylate linkers during PSE. Kim et al. described PSE in UiO-66 for a selection of solvents $\left(\mathrm{H}_{2} \mathrm{O}, \mathrm{DMF}, \mathrm{MeOH}\right.$ and $\left.\mathrm{CHCl}_{3}\right)$, resulting in different linker exchange yields. ${ }^{13,18}$ In the reports cited above, both defect generation plus healing as well as direct linker replacement are suggested as PSE mechanisms.

With some exceptions, ${ }^{17}$ the PSE studies reported thus far focused on comparing the parent material and the exchanged samples, with little insight into the process at the molecular level. Therefore, many questions regarding the ligand exchange mechanism remain unanswered, including its kinetics, the changes in the cluster coordination, the ratio of outgoing and incoming linkers, as well as the role of framework defects and solvent interactions. Nevertheless, as the examples above illustrate, ${ }^{13}$ parameters such as solvent composition clearly play a determining role in the exchange process. ${ }^{12}$ To address the remaining questions, we studied PSE in UiO-66 via time-resolved monitoring of both the exchange solution and the modified MOF materials. The combination of this approach with computational modeling and spectroscopic techniques provides direct insight in the ligand exchange mechanism and the parameters determining its outcome.

\section{EXPERIMENTAL DETAILS}

Synthesis of UiO-66 and ligand exchange. Reference UiO-66 material was prepared using a solvothermal protocol reported to afford materials with the near-perfect coordination of 12 BDC linkers per cluster, further referred to as 'modulator-free synthesis'.19 This material was activated through several solvent washing steps in DMF and ethanol (EtOH), followed by drying under dynamic vacuum at $473 \mathrm{~K}$ for $24 \mathrm{~h}$. After drying, this reference UiO-66 material was found to have $\mathbf{1 1 . 8}$ linkers per cluster. The BET surface area determined by $\mathrm{N}_{2}$ physisorption was found to be $1273 \mathrm{~m}^{2} \mathrm{~g}^{-1}$. The particle size was approximately $500 \mathrm{~nm}$, based on electron micrographs. The ligand exchange procedure followed that of Kim et al. ${ }^{12}: 28 \mathrm{mg}$ of UiO66 (containing $0.1 \mathrm{mmol}$ of BDC) was suspended in a solution of $18 \mathrm{mg}(0.1 \mathrm{mmol})$ of 2-aminoterephthalic acid $\left(\mathrm{NH}_{2}-\mathrm{H}_{2} \mathrm{BDC}\right)$ in $10 \mathrm{~mL}$ of methanol $(\mathrm{MeOH})$ at $313 \mathrm{~K}$. Under these conditions, equal amounts of both linkers are present in the mixture. The concentration of $10 \mathrm{mM}$ was selected to not exceed the experimentally determined solubility limit of either linker, namely 12 and $18 \mathrm{mM}$ in $\mathrm{MeOH}$ at room temperature for $\mathrm{H}_{2} \mathrm{BDC}$ and $\mathrm{NH}_{2}-\mathrm{H}_{2} \mathrm{BDC}$, respectively.

Time-resolved PSE monitoring. HPLC monitoring of the exchange solution was performed by injecting $1 \mu \mathrm{L}$ of sample on an Agilent $1260 \mathrm{HPLC}$ with a $1 \mathrm{~mL}$ min $^{-1}$ eluent flow (95:5 water/acetonitrile isocratic mixture) and equipped with a ZORBAX Eclipse XDB C18 column and a DAD detector tuned to the maximum absorbance wavelength of $240 \mathrm{~nm} \cdot{ }^{20}$ Analysis of the exchange solution confirmed the absence of side reactions during ligand exchange (e.g., esterification). The solid phase characterization was adapted from Shearer et al. ${ }^{21}$ and complemented via TGA and ${ }^{1} \mathrm{H}$ NMR analysis of the number of linkers per cluster, a measure of defect density. TGA analysis under $\mathrm{O}_{2}$ yields a quantitative measure of the organic/inorganic ratio in the framework by assuming full conversion to $\mathrm{ZrO}_{2}$ upon decomposition. Quantitative solution ${ }^{1} \mathrm{H}$ NMR of acid-digested MOF samples enables quantitative identification of the organic composition of the framework. Solution NMR and HPLC were both calibrated using standard solutions of $\mathrm{H}_{2} \mathrm{BDC}$ and $\mathrm{NH}_{2}-\mathrm{H}_{2} \mathrm{BDC}$. Reproducibility was assessed through triplicate trials, revealing standard deviations of 0.46-0.78, 0.28-0.89 and 0.49-1.2 linkers/cluster for HPLC, NMR and TGA, respectively. Further details on the experimental and characterization methods are provided in the Supporting Information (section SIII).

Computational methodology. Defective structure models were obtained from De Vos et al. ${ }^{22}$ by removal of linkers from the UiO-66 unit cell containing four $\left[\mathrm{Zr}_{6}\left(\mu_{3}-\mathrm{O}\right)_{4}\left(\mu_{3}-\mathrm{OH}\right)_{4}\right]^{12+}$ clusters. $^{1}$ A low number of defects is represented by a unit cell with one missing linker, resulting in two 12-fold and two 11-fold coordinated clusters. To model a higher number of defects, three linkers are removed, resulting in an average of 10.5 linkers per cluster. Of the many possible ways to remove three linkers, ${ }^{22,23}$ we chose two cases denoted as $(10 \mathrm{~b}, 10 \mathrm{~b}, 11,11)_{334}$ and $(9 \mathrm{c}, 11$, $11,11)_{333}$, where the numbers indicate the connectivity of each brick and the subscripts are determined by the symmetry of the unit cell. ${ }^{23}$ In the first case, two clusters are 10-fold coordinated and two are 11-fold coordinated. In the second, one 9-fold and three 11-fold coordinated clusters are present. A representation of the unit cells is given in Figure 2 . With each removed linker, four $\mathrm{Zr}$ atoms become 
coordinatively unsaturated and two positive charges on the inorganic node need to be compensated.

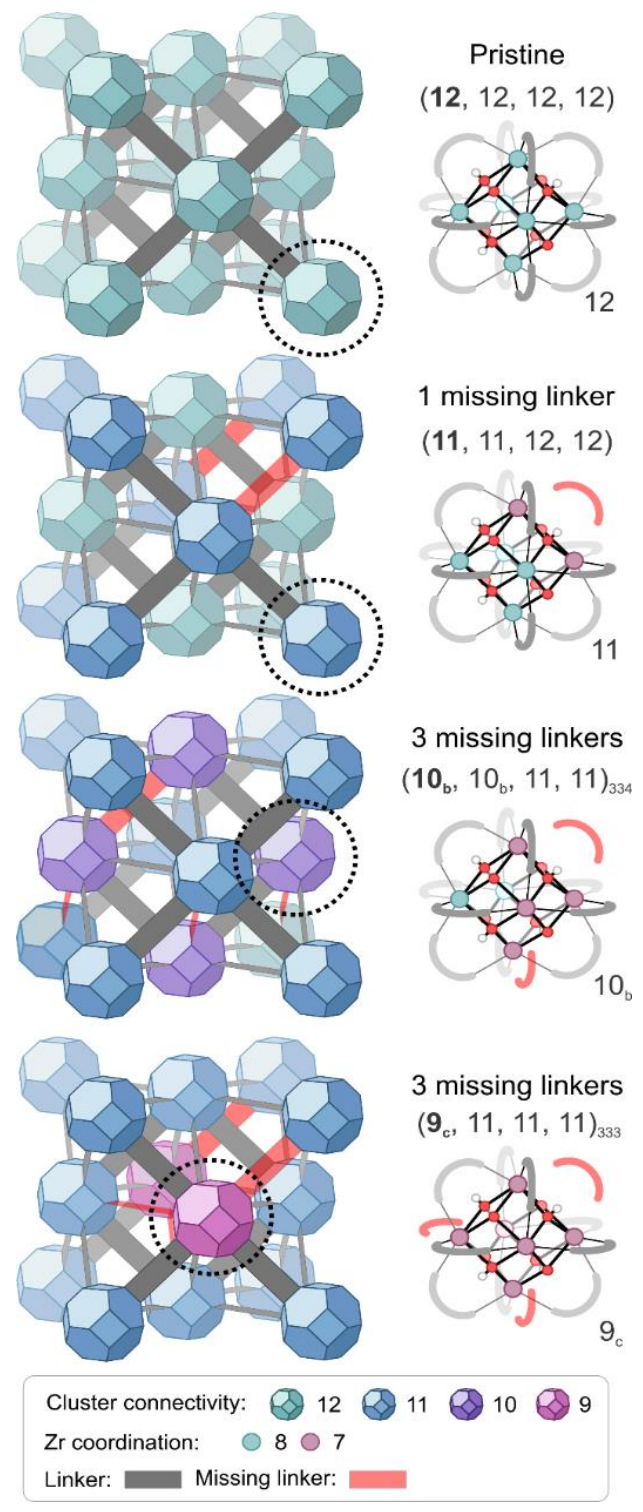

Figure 2. Structural models of defect-free and defective UiO-66 Left: the four UiO-66 unit cells used for computational modeling, with the missing linkers indicated in red. For clarity, the clusters and linkers at the border of the unit cell are represented in their entirety. Right: the four clusters that make up the unit cells, with connectivity ranging from 12 to 9 .

Charges at the defect sites can be compensated by adsorption of a negative species or removal of a proton. However, the latter configurations are higher in energy. ${ }^{24,25}$ In our models, the $\mathrm{Zr}$ atoms at the defect sites are capped by species likely present in the pores: $\mathrm{H}_{2} \mathrm{O}, \mathrm{OH}^{-}, \mathrm{MeOH}, \mathrm{MeO}^{-}$and $\mathrm{HCOO}^{-}$.
Taking into account the symmetry of the unit cell, multiple positions of the charge-compensating species were compared. All calculations were performed with periodic density functional theory (DFT) to fully represent the crystal environment. We made use of the periodic VASP code (Vienna Ab Initio Simulation Package), ${ }^{26-30}$ applying the projector augmented wave (PAW) approach ${ }^{31}$ and $\Gamma$-point approximation at PBE level of theory ${ }^{32}$ including GrimmeD3 dispersion corrections. ${ }^{33,34}$ The kinetic energy cutoff for the plane waves was set to $700 \mathrm{eV}$, and the convergence threshold for the electronic self-consistent field ( $\mathrm{SCF}$ ) calculations was fixed to $10^{-5} \mathrm{eV}$. A Gaussian smearing of $0.025 \mathrm{eV}$ was included to improve convergence. The volume for each unit cell (Figure 2) was optimized by fitting a Birch-Murnaghan equation of state. In the calculation of energies, to account for the finite temperature, thermal corrections were performed using the calculated frequencies. Enthalpic and entropic contributions were calculated with the TAMkin processing toolkit. 35 A partial Hessian vibrational analysis approach was used ${ }^{36}$ as this does not substantially affect entropy and enthalpy differences.

\section{RESULTS AND DISCUSSION}

\section{Monitoring the PSE process}

By monitoring both the MOF and the exchange medium over time, we aimed at filling in the remaining gaps in the understanding of PSE in UiO66. To facilitate data interpretation, linker concentrations in both the liquid and solid phases are reported as the number of BDC or $\mathrm{NH}_{2}-\mathrm{BDC}$ carboxylate groups per cluster, i.e. 12 in the fully connected, defect-free UiO-66 network. Uptake of $\mathrm{NH}_{2}-\mathrm{H}_{2} \mathrm{BDC}$ from and release of $\mathrm{H}_{2} \mathrm{BDC}$ into the exchange medium was monitored by HPLC (Figure 3, top). The most pronounced changes are observed in the initial $8 \mathrm{~h}$ of exchange, followed by equilibration. After $72 \mathrm{~h}$ of PSE, a BDC: $\mathrm{NH}_{2}-\mathrm{BDC}$ ratio of $0.9: 1$ is observed in the framework, indicating a slight preference for the latter linker. Although the composition of the framework changes dramatically during PSE, the UiO-66 crystallinity is preserved throughout the treatment as shown by PXRD (Figure S8). To exclude crystal dissolution and recrystallization, control experiments with mixtures of linker and $\mathrm{ZrCl}_{4}$ in $\mathrm{MeOH}$ in the concentration ranges relevant to PSE were performed (Table S2). No precipitate was formed with either linker after incubation at the exchange temperature. Additionally, UiO-66 crystals before and after exchange show no variation in particle size or shape (Figure S10). 

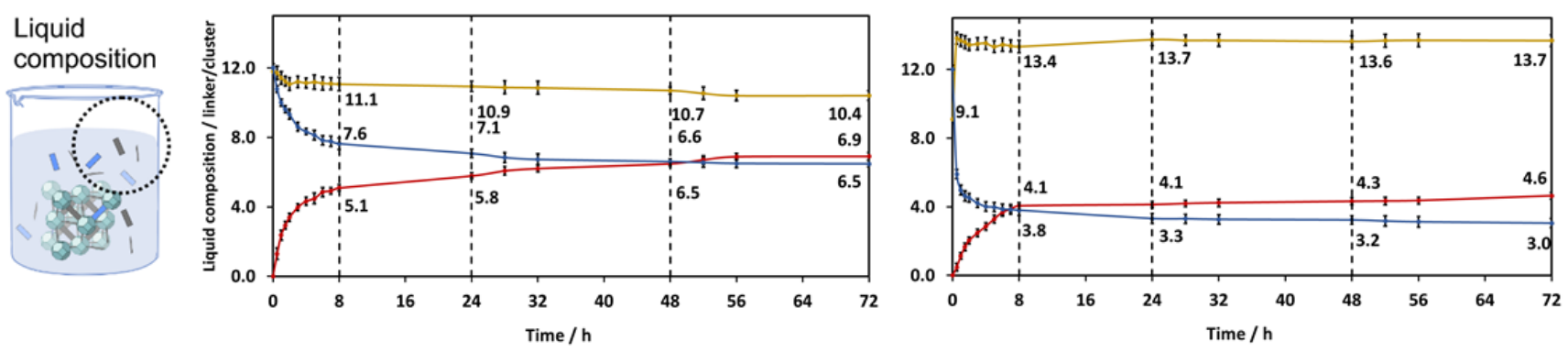

$\rightarrow$ Linker total (HPLC) $\rightarrow$ BDC (HPLC) $\rightarrow-\mathrm{NH}_{2}-\mathrm{BDC}$ (HPLC)
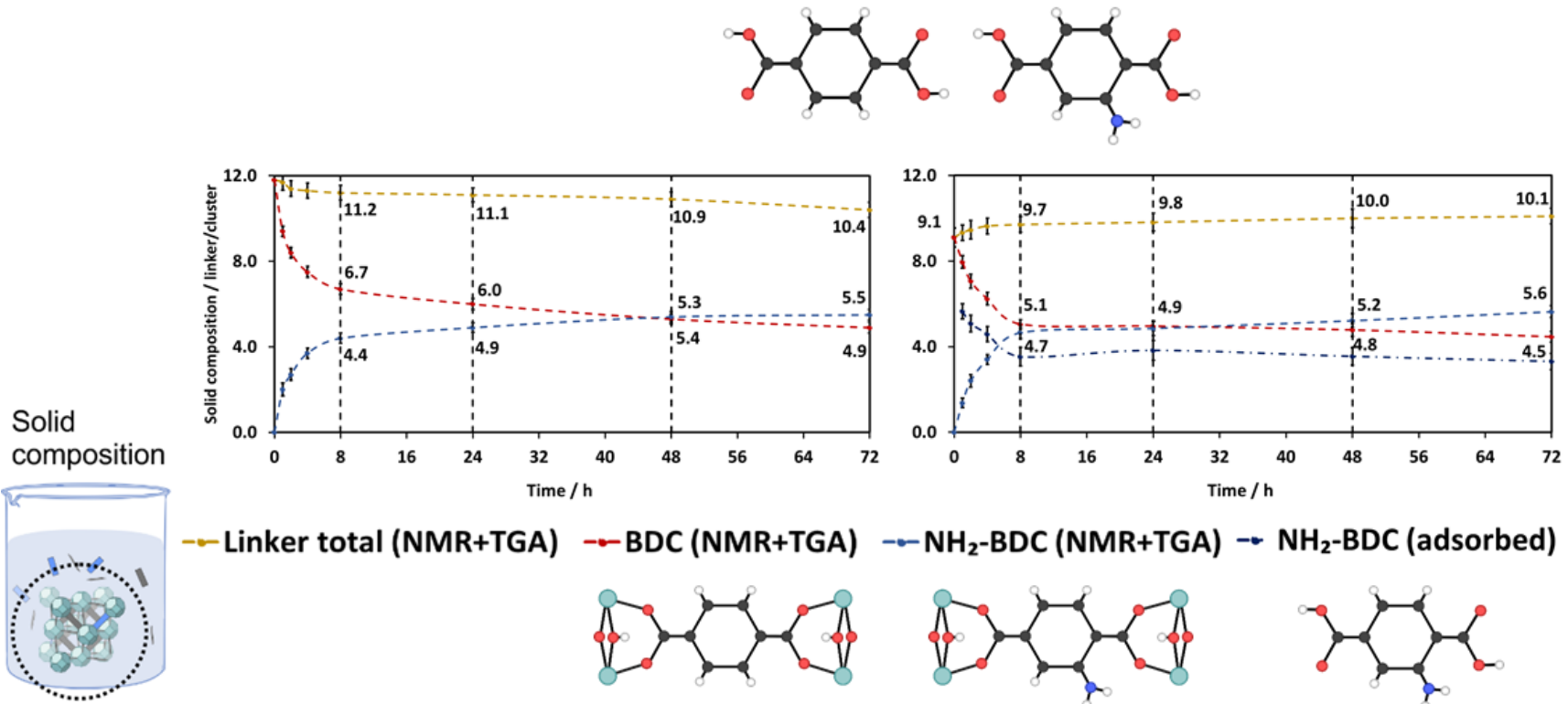

- - Linker total (NMR+TGA) $\quad-$ - BDC (NMR+TGA)

$\sim \mathrm{NH}_{2}-\mathrm{BDC}(\mathrm{NMR}+\mathrm{TGA}) \rightarrow \mathrm{NH}_{2}-\mathrm{BDC}$ (adsorbed)
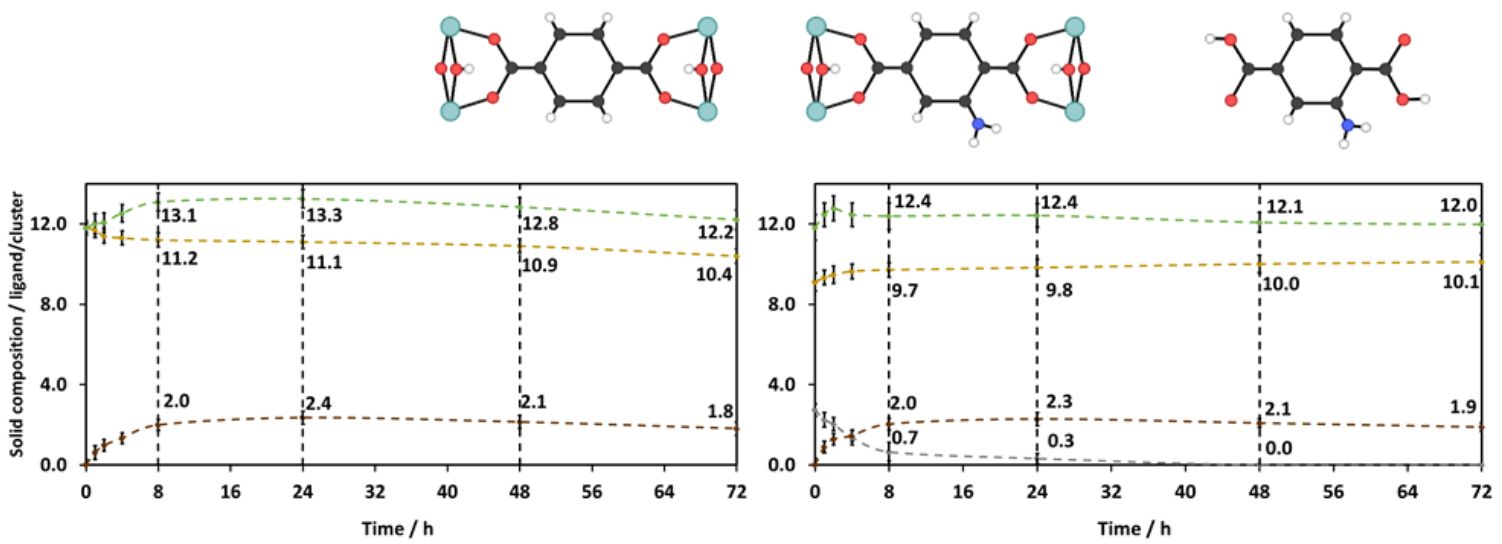

- - Ligand total (solid) - - Linker total (NMR+TGA)

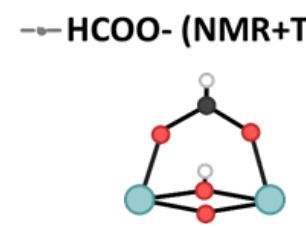

- $\mathrm{MeO}^{-}(\mathrm{NMR}+\mathrm{TGA})$

Figure 3. Time-resolved monitoring of PSE in defect-free UiO-66 (left) and defect-rich UiO-66 (right). Top: Exchange solution composition as determined by $\mathrm{HPLC}$, with $\mathrm{H}_{2} \mathrm{BDC}$ and $\mathrm{NH}_{2}-\mathrm{H}_{2} \mathrm{BDC}$ concentrations (expressed per inorganic cluster) shown in red and blue, respectively. The total number of linkers in the solid (yellow) is calculated based on the difference between BDC coming out and $\mathrm{NH}_{2}$-BDC going in. Middle: Linker content of the MOF material during PSE as determined by analysis of the solid by TGA and quantitative ${ }^{1} \mathrm{H}$ NMR, of acid 
digested samples The measured $\mathrm{H}_{2} \mathrm{BDC}$ and $\mathrm{NH}_{2}-\mathrm{H}_{2} \mathrm{BDC}$ contents are shown in dashed red and blue, respectively; the calculated number of linkers per cluster in the solid in dashed yellow. Bottom: The $\mathrm{MeO}^{-}$and $\mathrm{HCOO}^{-}$content in the MOF material during PSE, as determined by TGA and quantitative ${ }^{1} \mathrm{H}$ NMR, of acid digested samples, are shown in dashed brown and dashed grey, respectively. The total number of ligands per cluster (dashed green) is calculated as the sum of the linkers (dashed yellow, same curve as in middle panel) and the $\mathrm{MeO}^{-}$content. The NMR, TGA and HPLC measurements at each time instance were performed in triplicate. The error bars are based on these nine data points. The analysis results are presented in more detail in Figures S9 and $\mathrm{S} 13$.

In contrast to the PSE behavior commonly assumed, close analysis of the HPLC data shows nonstoichiometric linker exchange: more $\mathrm{H}_{2} \mathrm{BDC}$ is released than $\mathrm{NH}_{2}-\mathrm{H}_{2} \mathrm{BDC}$ is incorporated. Since the exchange conditions avoid solubility issues of either linker, these measurements indicate an actual decrease from 11.8 to 10.4 linkers per cluster during PSE, for the pristine UiO-66. To cross-check these observations with the changes in the solid phase, MOF samples were collected at selected instances and characterized for composition and structure (Figure 3, middle and bottom). Analysis of the linker ratio was performed via quantitative solution ${ }^{1} \mathrm{H}$ NMR after extensive washing with EtOH. The total organic content was determined by TGA and used as a reference for the NMR measurements. The solid phase data mirrors the observations from the solution data, with the most pronounced changes to the framework composition in the initial $8 \mathrm{~h}$. Moreover, these results reveal the same decline in the number of linkers per cluster as determined by HPLC, consistent with the generation of defects in the UiO-66 treated samples. As defects are necessarily formed by the net removal of linkers, negatively charged species different from BDC or $\mathrm{NH}_{2}$-BDC have to be incorporated in the framework. A clear distinction is made between linkers and ligands: the former term addresses the structural terephthalates that build up the framework, the latter includes all cluster-bound species, including terephthalates as well as other charged organic species responsible for charge compensation. To facilitate data interpretation, linker and ligand concentrations are both reported on a per-cluster basis.

A closer look at the NMR spectra of PSE UiO-66 samples digested with $\mathrm{CsF}$ and $\mathrm{DCl}$ in DMSO-d6, reveals a characteristic $\mathrm{MeOH}$ signal. Given that all samples were extensively washed with EtOH and vacuum dried at temperatures exceeding $373 \mathrm{~K}$ before analysis, this observation indicates a strong interaction between $\mathrm{MeOH}$ solvent species and the inorganic clusters. Since no EtOH could be detected, the two alcohols seem to have a different affinity for UiO-66. To better assess the effect of $\mathrm{MeOH}$, pristine UiO-66 was suspended in pure $\mathrm{MeOH}$ at the same temperature as used for PSE. Combined TGA analysis showed the loss of approximately one BDC linker per cluster, accompanied by an increasing MeOH NMR signal for acid-digested samples (Figure S6). Interestingly, Shearer et al. previously reported a decrease in linker content for UiO-66 washed with $\mathrm{MeOH} .{ }^{19}$ Recently, the coordination of $\mathrm{MeO}^{-}$to the $\left[\mathrm{Zr}_{6}\left(\mu_{3}-\mathrm{O}\right)_{4}\left(\mu_{3}-\mathrm{OH}\right)_{4}\right]^{12+}$ clusters was proposed by Yang et al. as a mechanism to stabilize missing linker defects. ${ }^{37}$ These observations indicate that even under mild conditions $\mathrm{MeOH}$ generates defects, most likely of the missing linker type, in the otherwise robust UiO-66 framework.

Adding monocarboxylic acids as 'modulators' is a common strategy to control the particle size or defectivity of Zr-based MOFs. ${ }^{38}$ In UiO-66, part of these monocarboxylates end up in the framework where they form missing linker and/or missing cluster defect sites that might affect PSE. ${ }^{16,17}$ To study the effect of such sites, defect-rich UiO-66 was prepared solvothermally in the presence of formic acid. The inorganic clusters in the resulting material are coordinated on average by 9.1 BDC linkers and 2.7 formate ligands

The material showed a slightly larger average particle size than the defect-free UiO-66 prepared in the absence of a modulator ( $2 \mu \mathrm{m}$ versus $500 \mathrm{~nm}$ ). As expected from the presence of defect sites, an increased BET surface area was measured, $1538 \mathrm{~m}^{2}$ $\mathrm{g}^{-1}$ compared to $1273 \mathrm{~m}^{2} \mathrm{~g}^{-1} .^{21}$ When subjected to linker exchange, the defective material shows a rapid change in composition in the first $8 \mathrm{~h}$ followed by equilibration, similar to the reference UiO-66 material (Figure 3, top and middle). Solid phase analysis shows that $\mathrm{HCOO}^{-}$is rapidly removed from the framework and can no longer be detected after $48 \mathrm{~h}$ (Figure 3, bottom, dashed grey line). At the same time, $\mathrm{MeOH}$ is incorporated, likely in the form of $\mathrm{MeO}^{-}$for charge compensation, at defect sites previously capped by $\mathrm{HCOO}^{-}$. However, after PSE, this $\mathrm{MeO}^{-}$content is lower than the amount of $\mathrm{HCOO}^{-}$ initially present (1.9 versus 2.7 per cluster), which hints at healing of a fraction of the defects (vide infra). 

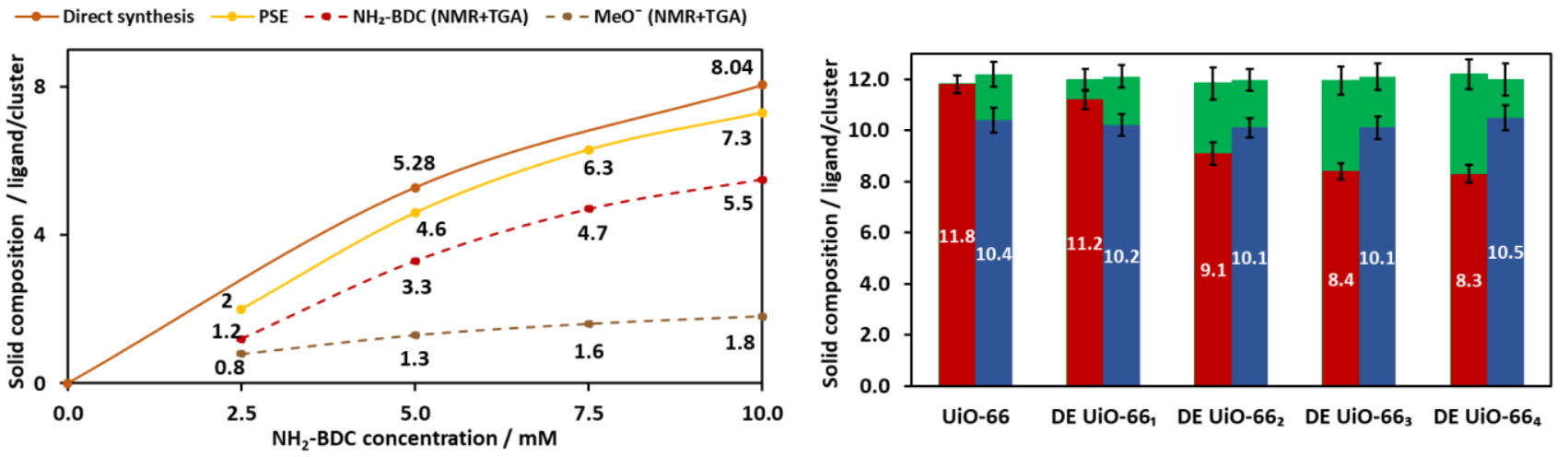

Figure 4. The equilibrium nature of the PSE process in MeOH. Left: Cluster coordination as a function of the $\mathrm{NH}_{2}$-BDC fraction offered either in modulator-free, direct synthesis or PSE ( $72 \mathrm{~h}$ in $\mathrm{MeOH}$ ). For the direct synthesis case, the number of $\mathrm{NH}_{2}$-BDC linkers per cluster is indicated (orange full line). For the PSE case, the sum of the $\mathrm{NH}_{2}$-BDC linkers and the $\mathrm{MeO}^{-}$ligands is indicated (yellow full line). Right: The effect of PSE on cluster coordination for UiO-66 materials with different initial defect densities. The 'defect-engineered' samples are indicated with the prefix 'DE'. The materials termed 'UiO-66' and 'DE UiO-662', correspond to the MOFs studied in Figure 3. The number of structural linkers per cluster before and after PSE is indicated in red and blue, respectively. The total cluster coordination, including both structural terephthalates and other charged species, is indicated in green. After PSE, the height of the green bar corresponds to the amount of incorporated $\mathrm{MeO}^{-}$. Error bars are based on solid phase analyses performed in triplicate.

While PSE in $\mathrm{MeOH}$ differs in details for defect-free and defect-rich UiO-66, the evolution and outcome of the process seem to be very similar for both materials. An exchange of structural linkers is detected in both cases, in contrast to what is observed for PSE in DMF. ${ }^{17}$ After exchange, virtually the same numbers of dicarboxylate linkers per cluster are observed in the defective and nondefective material, 10.1 versus 10.4 , with a $\mathrm{BDC}: \mathrm{NH}_{2}-$ $\mathrm{BDC}$ ratio of 0.8 and 0.9 , respectively. Also approximately the same amount of $\mathrm{MeO}^{-}$is eventually incorporated, 1.9 versus 1.8 per cluster, respectively. These very similar compositions strongly hint at an equilibrium process at the inorganic cluster level. To further explore this hypothesis, PSE trials were conducted with different $\mathrm{NH}_{2}$-BDC concentrations, $2.5-10 \mathrm{mM}$, corresponding to a $\mathrm{NH}_{2}-\mathrm{BDC}$ :BDC linker ratio of 1:4 - 1:1. The PSE results for a defect-free starting material are summarized in Figure 4, left. For comparison, the composition of a UiO-66 obtained through direct, modulator-free synthesis with the same $\mathrm{NH}_{2}$ $\mathrm{BDC}$ : $\mathrm{BDC}$ ratios is given as well. As expected when the final composition would be equilibriumcontrolled, the materials obtained through these different preparation routes show a similar trend, with more $\mathrm{NH}_{2}$-BDC ending up in the framework when a higher concentration is present in the synthesis mixture or exchange solution. The difference in composition between both materials seems mainly due to the incorporation of $\mathrm{MeO}^{-}$ during PSE.

To further test the idea of PSE as an equilibrium process, the composition after linker exchange was compared for UiO-66 samples with different initial defect densities. Through the use of different monocarboxylic acid modulators and concentrations, these materials covered a broad range of defectivity. The number of linkers per cluster ranged from 11.2 to 8.3, with the rest of the cluster coordination completed by monocarboxylate ligands. Strikingly, however, when subjected to identical PSE conditions, all samples were found to contain approximately 10 linkers and $2 \mathrm{MeO}^{-}$per cluster (Figure 4, right). These near-identical compositions, irrespective of the initial framework defect density, underline the equilibrium nature of the PSE process in $\mathrm{MeOH}$.

\section{Nature of the defect sites in $\mathrm{MeOH}$}

To understand the nature of the defects created by the net linker loss during PSE, the stability of these sites capped with different species was computationally investigated and compared to the defect-free material. Apart from $\mathrm{MeOH}$ and $\mathrm{MeO}^{-}$, also $\mathrm{H}_{2} \mathrm{O}, \mathrm{OH}^{-}$and $\mathrm{HCOO}^{-}$were included due to their possible presence as a result of synthesis solvent contamination or decomposition. ${ }^{21}$ The calculated enthalpy differences (Figure 5a) indicate a clear preference for capping missing linker defects with a $\mathrm{MeOH} / \mathrm{MeO}$ - pair, in line with the previous report by Yang et al. ${ }^{37}$ Note that the protonated $\mathrm{MeOH}$ molecule is removed during vacuum-drying and only the $\mathrm{MeO}-$ species is detected in NMR analysis of digested samples. Entropic contributions and dynamic solvent effects may further play a role in the stabilization of both the defective and non-defective 
material.39 Furthermore, free energy calculations that take entropic effects into account indicate that the formation of $\mathrm{MeOH} / \mathrm{MeO}^{-}$terminated defects becomes more favorable at lower temperatures since the entropic penalty associated with adsorption decreases (Figure 5b). Since PSE is performed at much lower temperatures than UiO-66 synthesis, the formation of missing linker defects can be rationalized through this lower free energy penalty. Vice versa, this computational result agrees well with the experimentally observed preference for forming non-defective UiO-66 at high synthesis temperatures. ${ }^{19}$ Knowing the nature of the ligands that balance the charge of the inorganic clusters also provides insight in the connectivity of the linkers and clusters. Quantification of the structural linkers and other charged ligands (i.e., $\mathrm{BDC}, \mathrm{NH}_{2}-\mathrm{BDC}$ and $\mathrm{MeO}^{-}$) reveals that during $P S E$ there are more than the required 12 ligating groups present per cluster (Figure 3, bottom, green dashed line). Most likely, this apparent 'overcoordination' can be caused by the presence of dicarboxylic linkers that are connected only through one end to an inorganic cluster, leaving the other end dangling in the pore, thus not contributing to charge compensation.

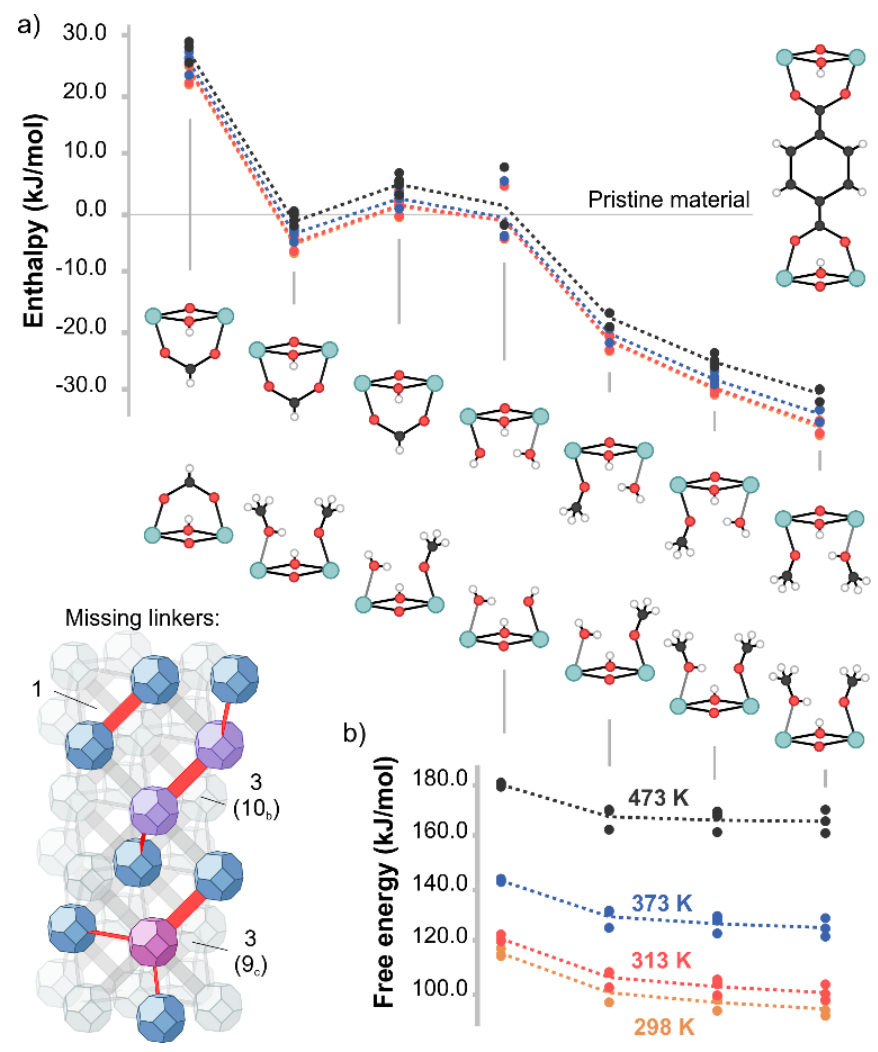

Figure 5. Energy diagrams for defective UiO-66 unit cells. Each dot represents a possible distribution of missing-linker defects ( 1 or 3 in total) within the unit cell; the connecting dotted line represents a weighted average (see Supporting Information). Values are normalized by the number of missing linkers in the unit cell. (a) Enthalpy difference between defect sites capped in different ways versus the non-defective material at $\mathrm{T}=298,313,373,473$ K. (b) Temperature-dependence of the free energy difference of the defective structures indicated above (capped with $\mathrm{H}_{2} \mathrm{O} / \mathrm{OH}^{-}, \mathrm{H}_{2} \mathrm{O} / \mathrm{MeO}^{-}$and $\mathrm{MeOH} / \mathrm{MeO}^{-}$) versus the non-defective structure. A representation of the clusters with different missing linker connectivities is also provided.

The framework composition reveals that this overcoordination is predominantly due to the incorporation of more than two MeO- groups per cluster (Figure 3, bottom). The total of over 13 ligands per cluster observed for pristine UiO-66 implies that on average each cluster has at least one dangling $\mathrm{BDC}$ or $\mathrm{NH}_{2}$-BDC linker connected to it. Interestingly, throughout the PSE process the degree of overcoordination first rises and then falls (Figure 3, bottom, green curve). Initially, the total number of ligands per cluster climbs to over 13 after $8 \mathrm{~h}$. Eventually, after $72 \mathrm{~h}$ of ligand exchange, almost no overcoordination is observed and approximately 12 ligands per cluster are present. This 2-step variation is counterintuitive and hints at the combined contribution of several processes occurring on a different timescale.

The lack of overcoordination at the end of the exchange process $(72 \mathrm{~h})$ indicates that there are few dangling linkers left. At the same time, $\mathrm{MeO}^{-}$species remain present in the structure (1.8 per cluster), presumably as $\mathrm{MeOH} / \mathrm{MeO}^{-}$pairs. Combined, these two observations imply that at the end of the PSE process most defects in the lattice take the form of a missing linker that has been replaced by two $\mathrm{MeOH} / \mathrm{MeO}^{-}$pairs directly opposite to each other, as displayed in Figure 5. In other words, the changes in overcoordination during $\mathrm{PSE}$ are due to $\mathrm{MeOH} / \mathrm{MeO}^{-}$ pairs, or alternatively the associated dangling linker sites, moving through the crystal lattice. As can be expected, this process is rather slow to come to an equilibrium. It was experimentally confirmed that longer PSE times do not further alter the framework composition. Based on the above observations, it can be hypothesized that (i) during PSE of UiO-66 in $\mathrm{MeOH}$ the solvent continuously generates dangling linker defects through the formation of $\mathrm{MeOH} / \mathrm{MeO}^{-}$ pairs and that (ii) eventually a state is reached in which $\mathrm{MeO}^{-}$ligands are still present, but in the form of double $\mathrm{MeOH} / \mathrm{MeO}^{-}$pairs, as indicated by the absence of dangling linkers.

\section{Monitoring the porosity throughout PSE}

Since dangling linkers affect the accessible pore volume, their appearance can be monitored through physisorption measurements. The BET surface areas determined for samples isolated at different moments during PSE show an abrupt drop in the 
initial $24 \mathrm{~h}$, from over $1200 \mathrm{~m}^{2} \mathrm{~g}^{-1}$ to below $600 \mathrm{~m}^{2} \mathrm{~g}^{-}$ ${ }^{1}$, followed by a gradual recovery to $1006 \mathrm{~m}^{2} \mathrm{~g}^{-1}$ (Figure 6, top). Composition analysis shows that the time instances where overcoordination is the highest coincide with the lowest specific surface area values. To further establish the relation between the measured surface area and the expected defect sites, theoretical BET surface areas were calculated for frameworks containing (i) dangling linkers and (ii) double $\mathrm{MeOH} / \mathrm{MeO}^{-}$pairs. Energy-optimized framework models with these defect sites are respectively thought of as representative of the intermediate regime with high overcoordination (and low surface area) and the final state without overcoordination (and partially recovered surface area). Zeo $++^{40}$ calculations estimate a surface area of $868 \mathrm{~m}^{2} \mathrm{~g}^{-1}$ for a UiO-66 material that contains on average one dangling BDC linker and one $\mathrm{MeOH} / \mathrm{MeO}^{-}$pair per cluster. Not surprisingly, this value is significantly lower compared to the theoretical surface area calculated for a defect-free UiO- 66 that would be similar to the starting material before PSE $\left(1147 \mathrm{~m}^{2} \mathrm{~g}^{-1}\right)$. Interestingly, when the dangling linker is replaced by a second $\mathrm{MeOH} / \mathrm{MeO}^{-}$ pair, the calculated surface area rises to $1057 \mathrm{~m}^{2} \mathrm{~g}^{-1}$. The correspondence between the calculated and measured surface areas, especially in combination with observed overcoordination, supports the hypothesis of the initial creation of dangling linkers, followed by the slow movement of defect sites.

In the defect-rich UiO-66 sample, no dangling linkers are observed (Figure 3, bottom, green dashed line). Together with the observation that the $\mathrm{MeO}^{-}$content after PSE is lower than the initial $\mathrm{HCOO}^{-}$content, this observation has two implications: (i) Part of the $\mathrm{HCOO}^{-}$initially present at defect sites is replaced by BDC or $\mathrm{NH}_{2}-\mathrm{BDC}$ structural linkers that are connected on both ends. It is our hypothesis that the extent of this 'healing', i.e. eliminating two adjacent monocarboxylate-capped sites in favor of a structural linker, corresponds to a small fraction of missing-linker defects that exists next to a larger number of missing-cluster sites; ${ }^{17}$ (ii) The sites adjacent to the predominant missing cluster defects are capped almost exclusively with $\mathrm{MeO}^{-} / \mathrm{MeOH}$. Likely, the high number of these 'edge' sites lining a defect causes the overcoordination during PSE to be much less pronounced than in the defect-free material where two $\mathrm{MeOH} / \mathrm{MeO}^{-}$pairs eventually seem to find a position directly opposing each other. Also in contrast to defect-free UiO-66, HPLC analysis of the PSE solution shows a considerably higher uptake of $\mathrm{NH}_{2}$-BDC than BDC release (Figure 3, top). However, this excess of $\mathrm{NH}_{2}-\mathrm{BDC}$ is not found when analyzing the solids after washing with EtOH. Given that the defective sample has a larger accessible pore volume because of missing cluster defects, ${ }^{21}$ this difference is likely due to weak $\mathrm{NH}_{2}-\mathrm{BDC}$ adsorption in solution. This hypothesis is supported by the drop in the adsorbed amount together with the replacement of $\mathrm{HCOO}^{-}$by the bulkier $\mathrm{MeOH} / \mathrm{MeO}^{-}$ pairs.

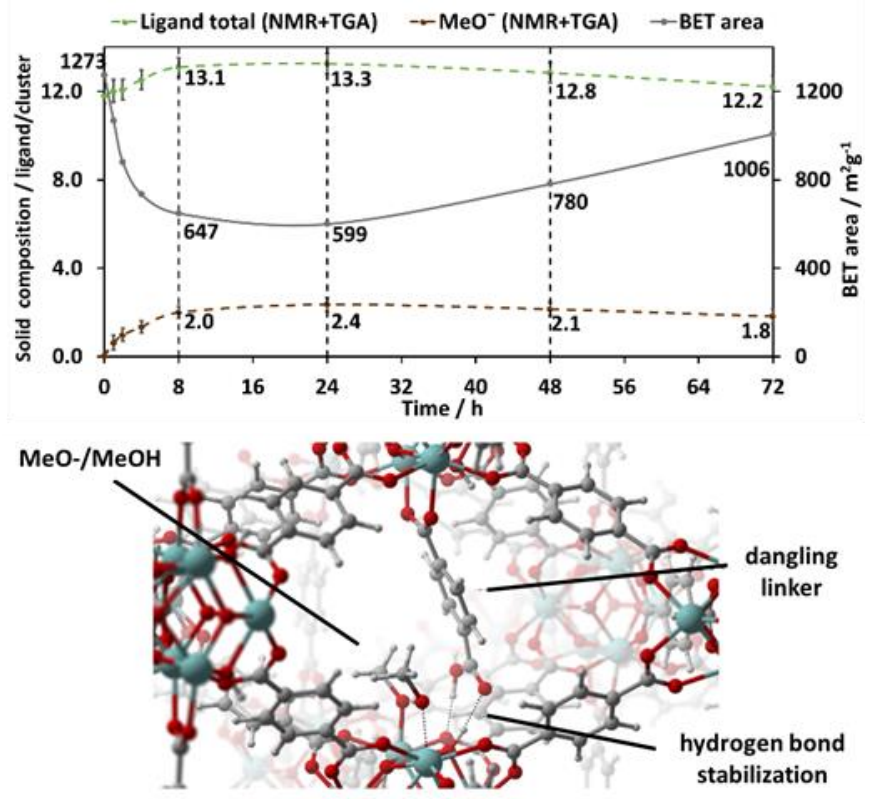

Figure 6. Porosity evolution during PSE of UiO-66 Top: $\mathrm{MeO}^{-}$content in the MOF material during PSE (dashed brown) as determined by NMR and TGA. The total number of ligands per cluster is shown in dashed green. The evolution of BET areas measured for samples collected at selected instances is shown in grey. Bottom: representation of an energy optimized UiO-66 structure with both dangling linkers and $\mathrm{MeO}^{-} / \mathrm{MeOH}$ pairs.

\section{Mechanism of PSE in $\mathrm{MeOH}$}

The exchange of structural linker, i.e. the release of BDC in favor of $\mathrm{NH}_{2}-\mathrm{BDC}$ incorporation, occurs a lot faster in $\mathrm{MeOH}$ compared to some other reported PSE conditions. A recent time-resolved study of PSE in DMF found that after $8 \mathrm{~h}$ at $323 \mathrm{~K}$ the defect-free UiO-66 starting material had lost less than $1 \%$ of the BDC linkers. ${ }^{17}$ In $\mathrm{MeOH}$ about $40 \%$ of the BDC originally present in the framework has been released and replaced by $\mathrm{NH}_{2}-\mathrm{BDC}$ (or $\mathrm{MeOH} / \mathrm{MeO}^{-}$ pairs) at the same point in time, even though both the exchange temperature and the concentration of incoming linker were lower than in the aforementioned study. Likely, the PSE process is greatly facilitated by the dangling linker states that are generated and stabilized in $\mathrm{MeOH}$ but not in solvents such as DMF. Only two Zr-O bonds need to be broken to remove a dangling linker from the framework, whereas exchanging a fully bound BDC would require breaking a total of four $\mathrm{Zr}-\mathrm{O}$ bonds, from two adjacent inorganic clusters. Indeed, under various PSE conditions, it has been observed that when monocarboxylates are present in the UiO-66 
starting material, these are exchanged faster than the structural linker. ${ }^{15,17,41-43}$ This preference is in line with static calculations, which show an enthalpy penalty of about $25 \mathrm{~kJ} / \mathrm{mol}$ per cluster for $\mathrm{HCOO}^{-}$ with respect to $\mathrm{BDC}$. Through the formation and stabilization of dangling states, $\mathrm{MeOH}$ enables the exchange of structural linkers with similar ease as for monocarboxylate ligands, an effect likely accredited to the solvent's acidic nature ( $\mathrm{pKa}=15.5)$ small kinetic diameter and hydrogen bonding capacity.

Non-defective material:

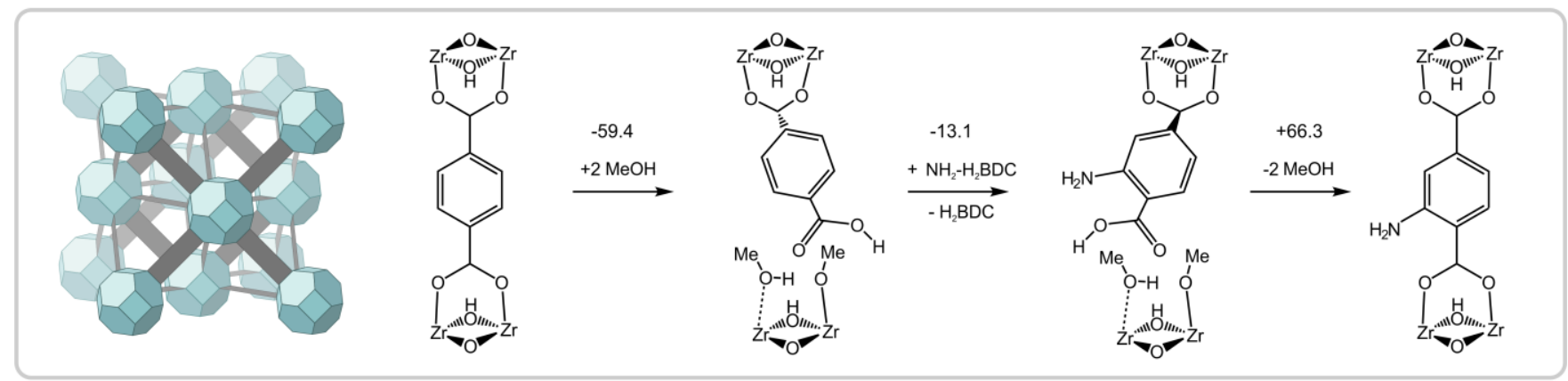

Defective material:

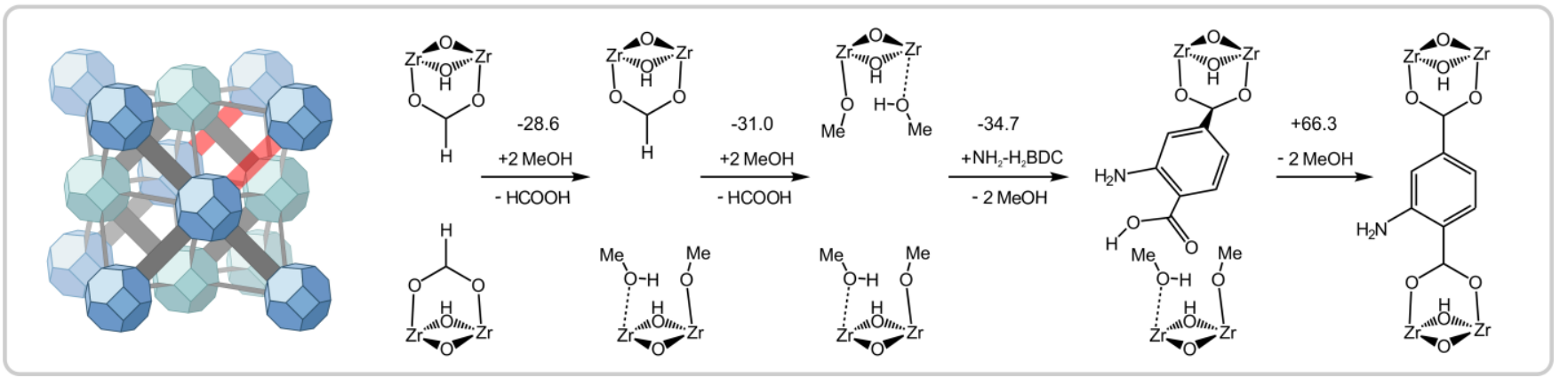

Figure 7. Proposed PSE mechanism in non-defective and defective UiO-66. MeOH facilitates ligand exchange through the creation and stabilization of defects. Enthalpy differences are given in $\mathrm{kJ} \mathrm{mol}^{-1}$ at $313 \mathrm{~K}(\mathrm{PSE}$ temperature).

Based on the above observations, a mechanism can be proposed for the ligand exchange of UiO-66 in $\mathrm{MeOH}$ and the relative enthalpies can be calculated for each involved state (Figure 7). The process is initiated by fast chemisorption of $\mathrm{MeOH}$ as $\mathrm{MeO}^{-}$, while a linker carboxylate group is protonated and detaches from the inorganic cluster. When $\mathrm{MeOH} / \mathrm{MeO}^{-}$pairs are formed at the defect site, this metastable dangling linker state is enthalpically favorable by $59.4 \mathrm{~kJ} \mathrm{~mol}^{-1}$ compared to the initial state. Hydrogen-bonding likely plays a key role in stabilizing the protonated linker, via the carboxylate oxygen atoms of other linkers and the cluster $\mu_{3}-\mathrm{OH}$ hydrogen. ${ }^{44}$ The dangling linker partially blocks the pore, as experimentally confirmed through an initially steeply decreasing BET surface area. Exchange of the mono-coordinated BDC linker with $\mathrm{NH}_{2}$-BDC follows, with partial coordination of the incoming linker. This process is considerably faster than exchange in DMF, in which the linkers remain fully coordinated. ${ }^{17} \mathrm{Next}, \mathrm{MeOH}$ is desorbed through re-protonation of the $\mathrm{MeO}^{-}$ligand and the coordination of $\mathrm{NH}_{2}$-BDC on both ends.

In defective UiO-66, the replacement of $\mathrm{HCOO}^{-}$with $\mathrm{MeOH} / \mathrm{MeO}^{-}$pairs results in an enthalpy decrease of $59.6 \mathrm{~kJ} \mathrm{~mol}^{-1}$ (Table $\mathrm{S} 3$ ). When $\mathrm{NH}_{2}-\mathrm{H}_{2} \mathrm{BDC}$ subsequently adsorbs at one of these defect sites, the cluster coordination becomes identical to the defectfree case. In both cases, dangling linkers in combination with $\mathrm{MeOH} / \mathrm{MeO}^{-}$sites continue to be created and the exchange process goes on until an equilibrium composition is achieved. The proposed re-protonation and partial decoordination of linkers throughout PSE is in complete agreement with a recent molecular dynamics study by some of the authors. ${ }^{4}$ Unlike DMF, MeOH likely facilitates the required proton transfer and hydrogen bond stabilization, in addition to its role in capping the resulting defects in an energetically favorable way. These insights in the mechanism rely on solvent-free periodic static calculations; it is likely that further stabilization is possible through additional hydrogen bonds with the solvent

Free energy calculations (Table S4-5) show that the 
PSE process is enthalpically driven. The net replacement of one BDC linker by $\mathrm{NH}_{2}$-BDC lowers the enthalpy of about $6.2 \mathrm{~kJ} \mathrm{~mol}^{-1}$. This slight preference is also observed in the equilibrium composition after both direct synthesis and PSE: more $\mathrm{NH}_{2}$-BDC than $\mathrm{BDC}$ ends up in the framework when stoichiometric quantities are present. Furthermore, the introduction of dangling or missing linker defects is more favorable at lower temperatures (Table S4-5). In other words, the UiO66 framework has a stronger preference for $\mathrm{MeOH} / \mathrm{MeO}^{-}$capped defects at the mild PSE temperature. PSE tests starting with different $\mathrm{NH}_{2}-$ BDC concentrations show that the higher the framework $\mathrm{NH}_{2}-\mathrm{BDC}$ content, the more $\mathrm{MeOH} / \mathrm{MeO}^{-}$ pairs are tolerated at equilibrium (SI 23). Calculations further show no substantial difference in enthalpy for unit cells with different amount of defects, in agreement with the experimental observations.

\section{Linker distribution after PSE}

The linker distribution after PSE has been the topic of some debate in literature, with some authors finding a homogeneous distribution while others observe a higher concentration of the incoming linker near the crystal surface. ${ }^{14,15,17,45}$ In the small UiO-66 particles studied here $(<1 \mu \mathrm{m})$, we expect defect generation by $\mathrm{MeOH}$, and therefore exchange, to occur homogeneously throughout the particle. To test this hypothesis, the linker distribution after 72 $\mathrm{h}$ of PSE was determined through solid-state NMR (SSNMR), by monitoring spin diffusion between chemically distinct ${ }^{1} \mathrm{H}$ nuclei. Since spin diffusion is governed by dipolar coupling, its rate is highly sensitive to the distance between the nuclei. ${ }^{46,47}$ After distinct NMR signals for the $\mathrm{NH}_{2}$-BDC and BDC protons were assigned (Figure 8, top), ${ }^{1} \mathrm{H}$ spin diffusion experiments were performed for different mixing times.

In larger particles, the finite diffusion rate of the incoming linker could, depending on the exchange rate, result in the formation of an enrichment near the crystal exterior. ${ }^{14,45}$ We used stimulated Raman scattering (SRS) microscopy to visualize the linker distribution in large UiO-66 crystals ( $>50 \mu \mathrm{m}$ ) after PSE in MeOH. SRS microscopy offers 3D vibrational imaging with a spatial resolution of $\sim 300 \mathrm{~nm} .{ }^{48,49}$ Large enough UiO-66 crystals were prepared by using formic acid as a modulator (DE UiO-664 ). The parent UiO-66 material shows a characteristic Raman signal at $1618 \mathrm{~cm}^{-1}$. The exchange of $\mathrm{NH}_{2}-\mathrm{BDC}$ into UiO-66 can be observed through the appearance of a band at $1592 \mathrm{~cm}^{-1}$, characteristic for UiO-66- $\mathrm{NH}_{2}$ (Figure 9, top). ${ }^{\circ}$ For the mixed-linker materials resulting from $\mathrm{PSE}$, the extent of $\mathrm{NH}_{2}-\mathrm{BDC}$ incorporation can be quantified by deconvolution of the spectra and taking the ratio of the signal intensities at 1592 and $1618 \mathrm{~cm}^{-1}\left(\mathrm{I}_{1592} / \mathrm{I}_{1618}\right)$. To validate this approach, the PSE process for the defect-rich UiO-66 crystals studied above (Figure 3) was monitored through SRS microscopy by measuring the average signal ratio at each time instance for a number of crystals.
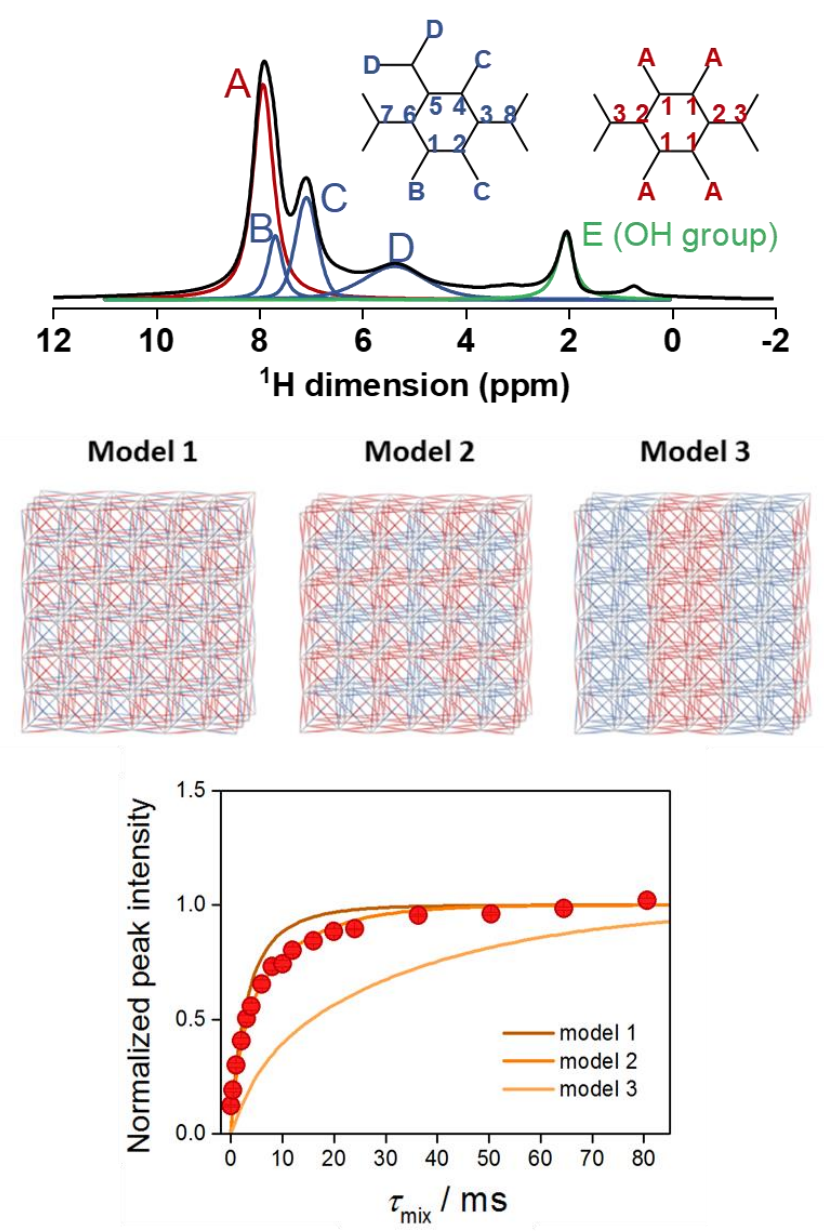

Figure 8. SSNMR analysis of the linker distribution after PSE. Top: SSNMR analysis of UiO-66 after PSE. ${ }^{1} \mathrm{H}$ MAS NMR spectrum with assignment of individual signals to the inequivalent hydrogen nuclei A-E. Middle: Mixed-linker models $(6 \times 6 \times 3$ unit cells $)$ with different degrees of linker mixing for a BDC: $\mathrm{NH}_{2}$ $\mathrm{BDC}$ ratio of $45: 55$. $\mathrm{BDC}$ and $\mathrm{NH}_{2}$ - $\mathrm{BDC}$ positions are indicated in red and blue, respectively. Bottom: SSNMR spin diffusion modeling. The calculated ${ }^{1} \mathrm{H}$ spin diffusion curves for the polarization transfer from protons $D$ to protons A are shown for each model with overlay of the experimental data.

The resulting profile closely resembles the evolution determined by the bulk analysis of the liquid and solid phase (Figure 9, middle). Next, the linker distribution in the large UiO-66 crystals was visualized by determining $\mathrm{I}_{1592} / \mathrm{I}_{1618}$ in a spatially resolved fashion. One of the strengths of SRS is that such imaging is performed in a confocal way and can therefore visualize a cross section through the UiO- 
66 crystal without potentially interfering contributions of the top and bottom planes. After 120 $\mathrm{h}$ of PSE, a cross sectional image taken at $30 \mu \mathrm{m}$ within the crystal clearly shows the presence of a gradient, with a gradually decreasing concentration of $\mathrm{NH}_{2}$-BDC towards the crystal interior. These results are in agreement with the observations by Matzger and co-workers for large UiO-66 crystals. ${ }^{14}$ We hypothesize that, in addition to diffusion effects, the dangling linkers intrinsic to PSE in $\mathrm{MeOH}$ further hamper the $\mathrm{NH}_{2}$-BDC transport, either through a lowered accessible pore volume or via hydrogen bonding interactions. Therefore, the $\mathrm{NH}_{2}-\mathrm{BDC}$ gradient can persist even long after equilibrium would be reached in smaller crystals $(<1-2 \mu \mathrm{m})$. Whether the linker distribution after PSE is homogeneous or not likely depends not only on crystal size, but might be solvent-dependent as well.
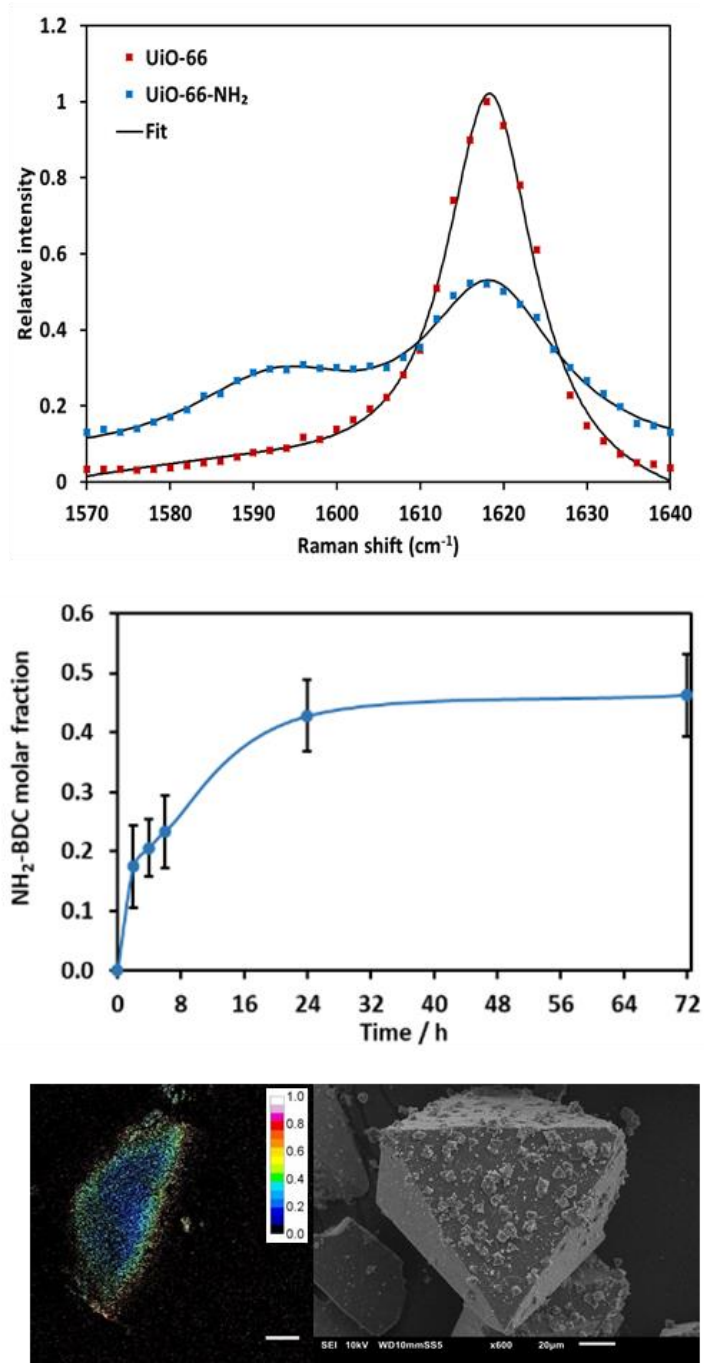

Figure 9. Monitoring of PSE through SRS microscopy. Top: SRS spectra of UiO-66 (red) and UiO-66- $\mathrm{NH}_{2}$ (blue). Middle: evolution of the $\mathrm{NH}_{2}$-BDC content for smaller UiO-66 crystals (1-2 $\mu \mathrm{m}$ diameter) as measured by the signal intensity ratio of the signals at $1592 \mathrm{~cm}^{-1}$ and $1618 \mathrm{~cm}^{-1}\left(\mathrm{I}_{1592} / \mathrm{I}_{1618}\right)$. The error bars are based on measurements of 5 crystals at each exchange time. Bottom: Confocal SRS cross section of a large UiO-66 crystal (100 $\mu$ m diameter) measured $30 \mu \mathrm{m}$ within the particle and a SEM micrograph of a crystal from the same batch. The intensity scale in confocal image corresponds to the ratio of the SRS signals at 1592 and $1618 \mathrm{~cm}^{-1}$. Exchange time: $120 \mathrm{~h}$. Scale bar: $10 \mu \mathrm{m}$.

\section{Conclusion}

In conclusion, we showed that time-resolved monitoring together with molecular modeling grants insight into PSE in UiO-66. The analysis of both solid and liquid phases grants for the first time unparalleled insight into the intricate nature of the interactions during PSE of UiO-66 in $\mathrm{MeOH}$. The active role of $\mathrm{MeOH}$ was revealed, in the first place in the creation and stabilization of dangling linker defects. Similarly to monocarboxylates, dangling linkers undergo fast exchange. The presence of missing linker or missing cluster defects at the start of the PSE process was shown to have no considerable impact on the equilibrium composition. After the exchange process, both linkers are distributed homogeneously in the framework for small UiO-66 crystallites. The current study represent a significant step forward in understanding the behavior of the UiO- 66 family and provides a reference for both rational design and applications of these and related materials.

\section{Suporting Information.}

Full analytic details including XRD patterns, SEM images, TGA curves, sorption isotherms, NMR spectra and all modelling results.

Acknowledgments

This project has received funding from the European Union's Horizon 2020 research and innovation program under the Marie Sklodowska-Curie (grant agreement No 641887, acronym: DEFNET). RA further acknowledges the funding from the European Research Council (grant agreement No 716472, acronym: VAPORE), as well as the Research Foundation Flanders (FWO) for funding in the projects Go83016N, 12R1217N and $1501618 \mathrm{~N}$. KU Leuven is acknowledged for funding in the project $\mathrm{C}_{32 / 16 / 019}$ VVS and JH further acknowledges financial support from the European Research Council (grant agreement Agency (research core funding No 647755, acronym: DYNPOR) and the Research Board of Ghent University (BOF). The computational resources and services used in this work were provided by the VSC (Flemish Supercomputer Center), funded by the FWO. AK and GM acknowledge the financial support from the 
Slovenian Research Agency (research core funding No. P1-0021 and project No. N1-0079). MR acknowledges financial support from the Research Foundation-Flanders (FWO, G.0962.13, AKUL/15/15 GoHo816N), KU Leuven Research Fund (C14/15/053, $\mathrm{OT} / 12 / 059)$, the Hercules foundation (HER/11/14), and funding from the European Research Council under the European Union's Seventh Framework Programme (FP/2007-2013)/ERC Grant Agreement no. [307523], ERC-Stg LIGHT to M.B.J.R.

\section{References}

(1) Cavka, J. H.; Jakobsen, S.; Olsbye, U.; Guillou, N.; Lamberti, C.; Bordiga, S.; Lillerud, K. P. A New Zirconium Inorganic Building Brick Forming Metal Organic Frameworks with Exceptional Stability. J. Am. Chem. Soc. 2008, 130 (42), 13850-13851.

(2) Wang, C.; Liu, X.; Keser Demir, N.; Chen, J. P.; Li, K. Applications of Water Stable Metal-Organic Frameworks. Chem Soc Rev 2016, 45 (18), 5107-5134.

(3) Van de Voorde, B.; Stassen, I.; Bueken, B.; Vermoortele, F.; De Vos, D.; Ameloot, R.; Tan, J.C.; Bennett, T. D. Improving the Mechanical Stability of Zirconium-Based Metal-Organic Frameworks by Incorporation of Acidic Modulators. J Mater Chem A 2015, 3 (4), 17371742.

(4) Vermoortele, F.; Bueken, B.; Le Bars, G.; Van de Voorde, B.; Vandichel, M.; Houthoofd, K.; Vimont, A.; Daturi, M.; Waroquier, M.; Van Speybroeck, V.; et al. Synthesis Modulation as a Tool To Increase the Catalytic Activity of Metal-Organic Frameworks: The Unique Case of UiO-66(Zr). J. Am. Chem. Soc. 2013, 135 (31), 11465-11468.

(5) Rogge, S. M. J.; Bavykina, A.; Hajek, J.; Garcia, H.; Olivos-Suarez, A. I.; Sepúlveda-Escribano, A.; Vimont, A.; Clet, G.; Bazin, P.; Kapteijn, F.; et al. Metal-Organic and Covalent Organic Frameworks as Single-Site Catalysts. Chem. Soc. Rev. 2017, 46 (11), 3134-3184.

(6) Wu, H.; Chua, Y. S.; Krungleviciute, V.; Tyagi, M.; Chen, P.; Yildirim, T.; Zhou, W. Unusual and Highly Tunable Missing-Linker Defects in Zirconium Metal-Organic Framework UiO-66 and Their Important Effects on Gas Adsorption. J. Am. Chem. Soc. 2013, 135 (28), 1052510532.

(7) Ren, J.; Langmi, H. W.; North, B. C.; Mathe, M.; Bessarabov, D. Modulated Synthesis of Zirconium-Metal Organic Framework (ZrMOF) for Hydrogen Storage Applications. Int. J. Hydrog. Energy 2014, 39 (2), 890-895.

(8) Stassen, I.; Bueken, B.; Reinsch, H.; Oudenhoven, J. F. M.; Wouters, D.; Hajek, J.; Van Speybroeck, V.; Stock, N.; Vereecken, P. M.; Van
Schaijk, R.; et al. Towards Metal-Organic Framework Based Field Effect Chemical Sensors: UiO-66- $\mathrm{NH}_{2}$ for Nerve Agent Detection. Chem Sci 2016, 7 (9), 5827-5832.

(9) Cunha, D.; Ben Yahia, M.; Hall, S.; Miller, S. R.; Chevreau, H.; Elkaïm, E.; Maurin, G.; Horcajada, P.; Serre, C. Rationale of Drug Encapsulation and Release from Biocompatible Porous Metal-Organic Frameworks. Chem. Mater. 2013, 25 (14), 2767-2776.

(10) Deria, P.; Mondloch, J. E.; Tylianakis, E.; Ghosh, P.; Bury, W.; Snurr, R. Q.; Hupp, J. T.; Farha, O. K. Perfluoroalkane Functionalization of NU-1000 via Solvent-Assisted Ligand Incorporation: Synthesis and $\mathrm{CO}_{2}$ Adsorption Studies. J. Am. Chem. Soc. 2013, 135 (45), 16801-16804.

(11) Deria, P.; Chung, Y. G.; Snurr, R. Q.; Hupp, J. T.; Farha, O. K. Water Stabilization of $\mathrm{Zr}_{6}$ Based Metal-Organic Frameworks via Solvent-Assisted Ligand Incorporation. Chem Sci 2015, 6 (9), 5172-5176.

(12) Kim, M.; Cahill, J. F.; Su, Y.; Prather, K. A.; Cohen, S. M. Postsynthetic Ligand Exchange as a Route to Functionalization of 'Inert' MetalOrganic Frameworks. Chem Sci 2012, 3 (1), 126-130.

(13) Kim, M.; Cahill, J. F.; Su, Y.; Prather, K. A.; Cohen, S. M. Postsynthetic Ligand Exchange as a Route to Functionalization of 'Inert' MetalOrganic Frameworks. Chem Sci 2012, 3 (1), 126-130.

(14) Boissonnault, J. A.; Wong-Foy, A. G.; Matzger, A. J. Core-Shell Structures Arise Naturally During Ligand Exchange in Metal-Organic Frameworks. J. Am. Chem. Soc. 2017, 139 (42), 14841-14844.

(15) Fluch, U.; Paneta, V.; Primetzhofer, D.; Ott, S. Uniform Distribution of Post-Synthetic Linker Exchange in Metal-Organic Frameworks Revealed by Rutherford Backscattering Spectrometry. Chem Commun 2017, 53 (48), 65166519.

(16) Shearer, G. C.; Vitillo, J. G.; Bordiga, S.; Svelle, S.; Olsbye, U.; Lillerud, K. P. Functionalizing the Defects: Post Synthetic Ligand Exchange in the Metal Organic Framework UiO-66. Chem. Mater. 2016, 28 (20), 7190-7193.

(17) Taddei, M.; Wakeham, R. J.; Koutsianos, A.; Andreoli, E.; Barron, A. R. Post-Synthetic Ligand Exchange in Zirconium-Based Metal-Organic Frameworks: Beware of the Defects! Angew. Chem. Int. Ed. 2018, 57 (36), 1170611710.

(18) Kim, M.; Cahill, J. F.; Fei, H.; Prather, K. A.; Cohen, S. M. Postsynthetic Ligand and Cation Exchange in Robust Metal-Organic Frameworks. J. Am. Chem. Soc. 2012, 134 (43), 18082-18088. 
(19) Shearer, G. C.; Chavan, S.; Ethiraj, J.; Vitillo, J. G.; Svelle, S.; Olsbye, U.; Lamberti, C.; Bordiga, S.; Lillerud, K. P. Tuned to Perfection: Ironing Out the Defects in Metal-Organic Framework UiO-66. Chem. Mater. 2014, 26 (14), 4068-4071.

(20) Liédana, N.; Lozano, P.; Galve, A.; Téllez, C.; Coronas, J. The Template Role of Caffeine in Its One-Step Encapsulation in MOF $\mathrm{NH}_{2}-\mathrm{MIL}-$ 88B(Fe). J Mater Chem B 2014, 2 (9), 11441151.

(21) Shearer, G. C.; Chavan, S.; Bordiga, S.; Svelle, S.; Olsbye, U.; Lillerud, K. P. Defect Engineering: Tuning the Porosity and Composition of the Metal-Organic Framework UiO-66 via Modulated Synthesis. Chem. Mater. 2016, 28 (11), 3749-3761.

(22) Rogge, S. M. J.; Wieme, J.; Vanduyfhuys, L.; Vandenbrande, S.; Maurin, G.; Verstraelen, T.; Waroquier, M.; Van Speybroeck, V. Thermodynamic Insight in the High-Pressure Behavior of UiO-66: Effect of Linker Defects and Linker Expansion. Chem. Mater. 2016, 28 (16), 5721-5732.

(23) De Vos, A.; Hendrickx, K.; Van Der Voort, P.; Van Speybroeck, V.; Lejaeghere, K. Missing Linkers: An Alternative Pathway to UiO-66 Electronic Structure Engineering. Chem. Mater. 2017, 29 (7), 3006-3019.

(24) Vandichel, M.; Hajek, J.; Ghysels, A.; De Vos, A.; Waroquier, M.; Van Speybroeck, V. Water Coordination and Dehydration Processes in Defective UiO-66 Type Metal Organic Frameworks. CrystEngComm 2016, 18 (37), 70567069 .

(25) Caratelli, C.; Hajek, J.; Cirujano, F. G.; Waroquier, M.; Llabrés i Xamena, F. X.; Van Speybroeck, V. Nature of Active Sites on UiO66 and Beneficial Influence of Water in the Catalysis of Fischer Esterification. J. Catal. 2017, 352, 401-414.

(26) Kresse, G.; Joubert, D. From Ultrasoft Pseudopotentials to the Projector Augmented-Wave Method. Phys. Rev. B 1999, 59 (3), 1758-1775.

(27) Kresse, G.; Furthmüller, J. Efficient Iterative Schemes for $A b$ Initio Total-Energy Calculations Using a Plane-Wave Basis Set. Phys. Rev. $B$ 1996, 54 (16), 11169-11186.

(28) Kresse, G.; Furthmüller, J. Efficiency of Ab-Initio Total Energy Calculations for Metals and Semiconductors Using a Plane-Wave Basis Set. Comput. Mater. Sci. 1996, 6 (1), 15-50.

(29) Kresse, G.; Hafner, J. Ab Initio Molecular-Dynamics Simulation of the Liquid-Metal-Amorphous-Semiconductor Transition in Germanium. Phys. Rev. B 1994, 49 (20), 1425114269.

(30) Kresse, G.; Hafner, J. Ab Initio Molecular Dynamics for Liquid Metals. 1993, 4.
(31) Blöchl, P. E. Projector Augmented-Wave Method. Phys. Rev. B 1994, 50 (24), 1795317979.

(32) Perdew, J. P.; Burke, K.; Ernzerhof, M. Generalized Gradient Approximation Made Simple. Phys. Rev. Lett. 1996, 77 (18), 3865-3868.

(33) Grimme, S. Accurate Description of van Der Waals Complexes by Density Functional Theory Including Empirical Corrections. J. Comput. Chem. 2004, 25 (12), 1463-1473.

(34) Grimme, S.; Antony, J.; Ehrlich, S.; Krieg, H. A Consistent and Accurate Ab Initio Parametrization of Density Functional Dispersion Correction (DFT-D) for the 94 Elements H-Pu. $J$. Chem. Phys. 2010, 132 (15), 154104.

(35) Ghysels, A.; Verstraelen, T.; Hemelsoet, K.; Waroquier, M.; Van Speybroeck, V. TAMkin: A Versatile Package for Vibrational Analysis and Chemical Kinetics. J. Chem. Inf. Model. 2010, 50 (9), 1736-1750.

(36) Ghysels, A.; Van Neck, D.; Waroquier, M. Cartesian Formulation of the Mobile Block Hessian Approach to Vibrational Analysis in Partially Optimized Systems. J. Chem. Phys. 2007, 127 (16), 164108.

(37) Yang, D.; Bernales, V.; Islamoglu, T.; Farha, O. K.; Hupp, J. T.; Cramer, C. J.; Gagliardi, L.; Gates, B. C. Tuning the Surface Chemistry of Metal Organic Framework Nodes: Proton Topology of the Metal-Oxide-Like $\mathrm{Zr} 6$ Nodes of UiO-66 and NU-1000. J. Am. Chem. Soc. 2016, 138 (46), 15189-15196.

(38) Trickett, C. A.; Gagnon, K. J.; Lee, S.; Gándara, F.; Bürgi, H.-B.; Yaghi, O. M. Definitive Molecular Level Characterization of Defects in UiO66 Crystals. Angew. Chem. 2015, 127 (38), 11314-11319.

(39) Caratelli, C.; Hajek, J.; Rogge, S. M. J.; Vandenbrande, S.; Meijer, E. J.; Waroquier, M.; Van Speybroeck, V. Influence of a Confined Methanol Solvent on the Reactivity of Active Sites in UiO-66. ChemPhysChem 2017, 19 (4), 420-429.

(40) Willems, T. F.; Rycroft, C. H.; Kazi, M.; Meza, J. C.; Haranczyk, M. Algorithms and Tools for High-Throughput Geometry-Based Analysis of Crystalline Porous Materials. Microporous Mesoporous Mater. 2012, 149 (1), 134-141.

(41) Shearer, G. C.; Chavan, S.; Bordiga, S.; Svelle, S.; Olsbye, U.; Lillerud, K. P. Defect Engineering: Tuning the Porosity and Composition of the Metal-Organic Framework UiO-66 via Modulated Synthesis. Chem. Mater. 2016, 28 (11), 3749-3761.

(42) Gutov, O. V.; Hevia, M. G.; Escudero-Adán, E. C.; Shafir, A. Metal-Organic Framework (MOF) Defects under Control: Insights into the Missing Linker Sites and Their Implication 
in the Reactivity of Zirconium-Based Frameworks. Inorg. Chem. 2015, 54 (17), 83968400 .

(43) Gutov, O. V.; Molina, S.; Escudero-Adán, E. C.; Shafir, A. Modulation by Amino Acids: Toward Superior Control in the Synthesis of Zirconium Metal-Organic Frameworks. Chem. Eur. J. 2016, 22 (38), 13582-13587.

(44) Hajek, J.; Caratelli, C.; Demuynck, R.; De Wispelaere, K.; Vanduyfhuys, L.; Waroquier, M.; Van Speybroeck, V. On the Intrinsic Dynamic Nature of the Rigid UiO-66 Metal-Organic Framework. Chem. Sci. 2018, 9 (10), 2723-2732.

(45) Jayachandrababu, K. C.; Sholl, D. S.; Nair, S. Structural and Mechanistic Differences in Mixed-Linker Zeolitic Imidazolate Framework Synthesis by Solvent Assisted Linker Exchange and de Novo Routes. J. Am. Chem. Soc. 2017, 139 (16), 5906-5915.

(46) Krajnc, A.; Kos, T.; Zabukovec Logar, N.; Mali, G. A Simple NMR-Based Method for Studying the Spatial Distribution of Linkers within Mixed-Linker Metal-Organic Frameworks. Angew. Chem. Int. Ed. 2015, 54 (36), 1053510538.

(47) Krajnc, A.; Bueken, B.; De Vos, D.; Mali, G. Improved Resolution and Simplification of the Spin-Diffusion-Based NMR Method for the Structural Analysis of Mixed-Linker MOFs. $J$. Magn. Reson. 2017, 279, 22-28.

(48) Freudiger, C. W.; Wei, M.; Saar, B. G.; Lu, S.; Holtom, G. R.; He, C.; Tsai, J. C.; Kang, J. X.; Xie, X. S. Label-Free Biomedical Imaging with High Sensitivity by Stimulated Raman Scattering Microscopy. Science 2008, 322, 1857.

(49) Liu, K.-L.; Kubarev, A. V.; Van Loon, J.; Uji-i, H.; De Vos, D. E.; Hofkens, J.; Roeffaers, M. B. J. Rationalizing Inter- and Intracrystal Heterogeneities in Dealuminated Acid Mordenite Zeolites by Stimulated Raman Scattering Microscopy Correlated with Super-Resolution Fluorescence Microscopy. ACS Nano 2014, 8 (12), 12650-12659.

(50) Otal, E. H.; Kim, M. L.; Calvo, M. E.; Karvonen, L.; Fabregas, I. O.; Sierra, C. A.; Hinestroza, J. P. A Panchromatic Modification of the Light Absorption Spectra of Metal-Organic Frameworks. Chem Commun 2016, 52 (40), 66656668 . 
For Table of Contents Only

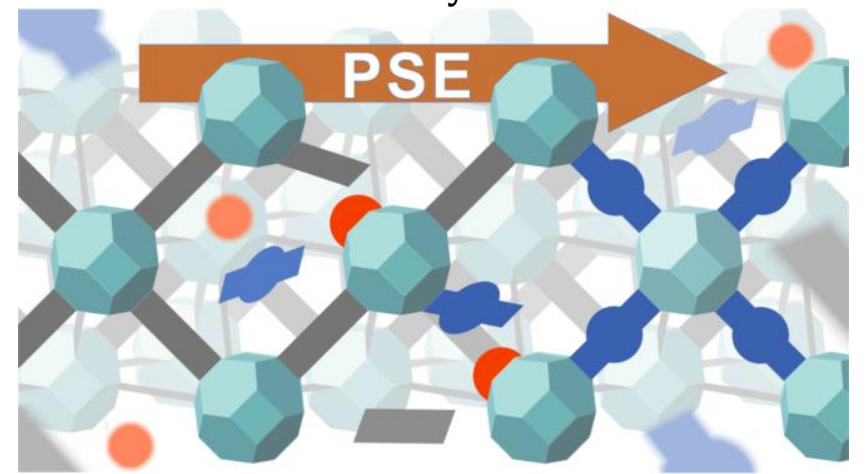

